Xiao Zhang, Yanlu Liu, Panfang Lu* and Min Zhang

\title{
Preparation and properties of hydrogel based on sawdust cellulose for environmentally friendly slow release fertilizers
}

https://doi.org/10.1515/gps-2020-0015

Received July 15, 2019; accepted October 30, 2019.

Abstract: A novel hydrogel slow-release nitrogen fertilizer based on sawdust with water absorbency was prepared using grafting copolymerization. Urea was incorporated as nitrogen source in a hydrogel fertilizer. Potassium persulfate (KPS) and N,N'-methylenebis acrylamide (MBA) were used as the initiator and crosslinker, respectively. The structure and properties of the samples were characterized by XPS, EDS, SEM, XRD and FTIR. The effects of various salt solutions, ionic strength and $\mathrm{pH}$ on swelling behavior were discussed. The results showed that the largest water absorbency of the sample reached $210 \mathrm{~g} / \mathrm{g}$ in distilled water. In addition, the sample had the good nitrogen release property. Thus, the novel environmentally friendly hydrogel fertilizer may be widely applied to agricultural and horticultural fields.

Keywords: sawdust; slow-release nitrogen fertilizer; water absorbency; swelling behavior; release behavior

\section{Introduction}

With the increase of the population, the world's population has reached 7.5 billion by 2017 [1]. The insufficient food has become a major problem for humanity, the most effective way to solve this problem is to increase grain production. Fertilizer and water are the most important

\footnotetext{
* Corresponding author: Panfang Lu, College of Chemistry and Material Science, Shandong Agricultural University, Tai'an, Shandong 271018, P. R. China, e-mail: xiaolu980216@163.com Xiao Zhang and Yanlu Liu, College of Chemistry and Material Science, Shandong Agricultural University, Tai'an, Shandong 271018, P. R. China

Min Zhang, National Engineering Research Center for Slow/ Controlled Release Fertilizers, National Engineering Laboratory for Efficient Utilization of Soil and Fertilizer Resources, College of Resources and Environment, Shandong Agricultural University, Tai'an, Shandong 271018, P. R. China
}

factors affecting agricultural production, so it is very important to improve utilization of fertilizer nutrient and water resources [2-4]. Urea is the most widely used nitrogen fertilizer which has a high nitrogen content and low production cost. However, urea released rapidly in a short time in soil [5-7]. Volatilization, leaching, and runoff of nutrients caused waste of fertilizers and contamination of water [8]. With the reduction of freshwater resources, irrigation water sources also are deficient. Therefore, it was very important to establish a reasonable water and fertilizer system.

The hydrogel is a superabsorbent polymer with a three-dimensional network structure. Hydrogel can absorb large amounts of liquids, which can weigh dozens or even hundreds of times their own weight [9]. Because of the strong water absorbency of hydrogel, it have been widely used to be water-retaining materials $[10,11]$. The hydrogel slow-release fertilizer (HSRF) is a multifunctional fertilizer, which can not only improve the utilization rate of fertilizer, and reduce the harm to the environment, but also maintain the soil moisture [12]. HSRF has excellent water retention, and effectively reduces irrigation frequency and production costs. At the same time, based on the slow release characteristics of HSRF, the mortality of plants reduced and the utilization rate of fertilizer was improved [13,14]. Fertilizer can be loaded into the hydrogel by many techniques, two methods were most commonly used: one is that the fertilizers are directly grafted on the hydrogel polymer chains, and the other one is that the hydrogel is first synthesized, and then the hydrogel is immersed in the fertilizers solution until it is saturated [14]. However, the application of hydrogel has encountered some problems in agricultural fields. Traditional hydrogel was synthesized from acrylic acid (AA) and acrylamide (AM). AA and AM were expensive and had poor degradation performance, which was not suitable for the sustainable development of green agriculture [15]. Therefore, some researchers have proposed to use bio-materials to synthesize hydrogel, such as starch [16,17], wheat straw [13,17,18], pineapple peel [19], leftover rice [20], corncob [8], flax yarn waste [9], chicken gelatin [21], yeast [22], methyl chitosan [23], cotton stalk 
[4,8], mulberry branch [24], cellulose acetate [25] and so on. Sawdust is a renewable and environmentally friendly biomass resource, which contain $61.2 \%$ cellulose [26-28]. However, most of sawdust was burnt, and the residual value of sawdust cannot be fully utilized [29]. As far as we know, there were no reports with the application of sawdust in hydrogel fertilizers fields. Therefore, we tried to use sawdust to prepare HSRF, thereby improving water absorption and fertilizer utilization to develop the environment-friendly agriculture.

The aim of this work is to synthesize a HSRF based on sawdust, AA, AM, and urea. The structure and properties of HSRF were characterized by using FTIR, XRD, SEM, XPS, EDS and TGA. In addition, water holding capacity, water absorbency capacity, nitrogen release behavior, swelling kinetics and release kinetics of the sample were also investigated systematically.

\section{Materials and methods}

\subsection{Materials}

Sawdust was provided by Sanyou Furniture Factory. Sodium hydroxide, N,N'-methylenebis acrylamide (MBA), potassium persulfate (KPS), hydrochloric acid and ethyl alcohol were obtained from Kaitong Chemical Reagent Co., Ltd. (Tianjin, China). Acrylic acid monomer (AA) and acrylamide (AM) were purchased from Macklin Chemical Reagents Co., Ltd. (Shanghai, China). Urea particles were obtained from Lanhua Coal Mining Group Co., Ltd. (Shanxi, China). Deionized water was used throughout the experiment. All reagents were of analytical reagent grade or commercial purity and were easily obtained from commercial sources.

\subsection{Preparation of sawdust cellulose (SC)}

First of all, the sawdust was washed and dried, then crushed and sieved (100 mesh). The sawdust powder was immersed in $10 \mathrm{wt} \%$ ammonia solution for $48 \mathrm{~h}$ and the mass ratio of sawdust powder to $10 \mathrm{wt} \%$ ammonia solution was 1:12, then the sawdust powder was filtered and washed with deionized water until the washing water was clean. Then, the sawdust powder was dried at $70^{\circ} \mathrm{C}$ in a drying oven. Next, the sawdust powder was put in $1 \mathrm{~mol} / \mathrm{L}$ nitric acid and boiled for $45 \mathrm{~min}$, and the mass ratio of sawdust to nitric acid was 1:12. And the sawdust powder was filtered and washed by using the same method until the $\mathrm{pH}$ of washing water was 7 . After the samples were dried, the sawdust cellulose were obtained [17].

\subsection{Preparation of HSRF}

$2 \mathrm{~g}$ sodium hydroxide was dissolved in $30 \mathrm{~mL}$ deionized water and the mixed solution was added to a $250 \mathrm{~mL}$ flask equipped with a mechanical stirrer. The flask was put in a water bath to make sure constant condition of $70^{\circ} \mathrm{C}$. Then, $6 \mathrm{~g} \mathrm{AA}, 3 \mathrm{~g}$ AM, $2 \mathrm{~g}$ urea and $0.07 \mathrm{~g}$ MBA were added to the flask. Next, flask was purged with nitrogen until the reaction completed. After stirring for $15 \mathrm{~min}$, the mixed solutions ( $30 \mathrm{~mL}$ deionized water, $1 \mathrm{~g}$ sawdust cellulose and $0.15 \mathrm{~g} \mathrm{KPS}$ ) were added into the above-mentioned flask. The water bath was kept at $70^{\circ} \mathrm{C}$ and stirred continuously for $4 \mathrm{~h}$ to complete the polymerization process. After the polymerization reaction completed, the sample was washed with ethanol to remove unreacted parts. At last, the HSRF was cut into small pieces $(2 \times 2 \mathrm{~mm})$ and placed in a drying oven at $70^{\circ} \mathrm{C}$ to constant weight and stored for future use. The mechanism of the polymerization is shown in Scheme 1. The preparation process of SC-g-PAA/ $\mathrm{PAM} /$ Urea is shown in Scheme 2.

\subsection{Characterization}

The FTIR spectra of samples were analyzed by singusing a Nicolet 380 FTIR spectrometer. The samples were subjected to wave numbers within the range of 500 to $4000 \mathrm{~cm}^{-1}$ at a resolution of $4 \mathrm{~cm}^{-1}$. The thermal stability of the various samples were evaluated under $\mathrm{N}_{2}$ circumstance by thermo-gravimetric analysis (TGA, DTG60A, Japan), the temperature was heated from $20^{\circ} \mathrm{C}$ to $800^{\circ} \mathrm{C}$ at a rate of $10^{\circ} \mathrm{C} / \mathrm{min}$. The morphologies of samples were analyzed by scanning electron microscopy (SEM, JSM-5800, Japan). The surface elemental composition and distribution of the samples were observed by X-ray photoelectron spectrometer (XPS, Escalab 250Xi, America) and Energy dispersive spectrometer (EDS). The X-ray diffracion(XRD) measurements were couducted by an X-ray diffractometer (XRD, D8 Advance, Germany).

\subsection{Swelling capacity and kinetics}

$0.2 \mathrm{~g}$ of the dried sample was immersed in $150 \mathrm{~mL}$ of deionized water and $\mathrm{NaCl}$ solution (1.0 wt\%), respectively, which were allowed to absorb solution until swelling 


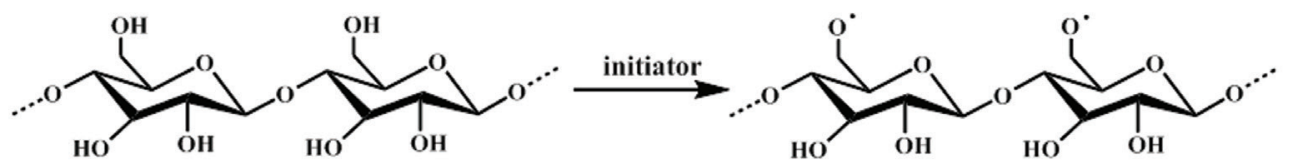<smiles>C=CC(=O)NCNC(=O)C=C</smiles>

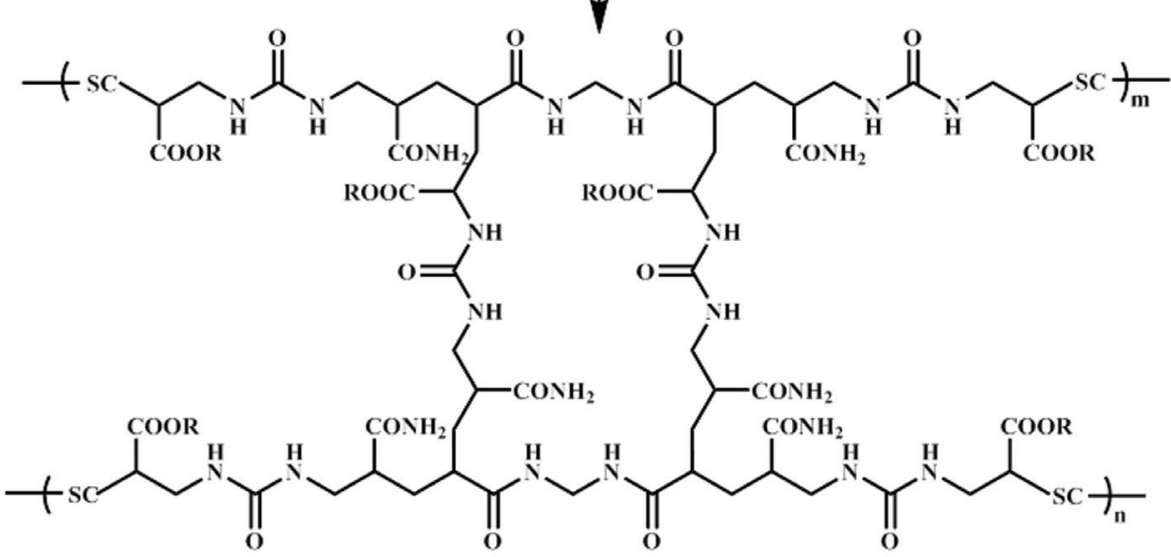

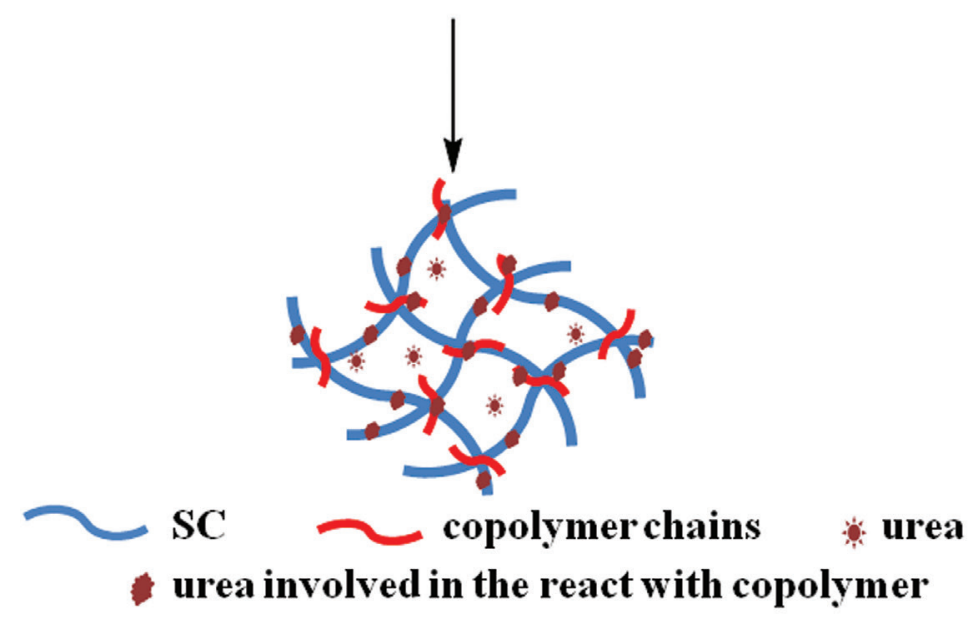

Scheme 1: The preparation scheme of SC-g-PAA/PAM/Urea.

equilibrium was obtained. Then, the swollen samples were quickly separated from the solution and measured the weight of the HSRF. The equilibrium water absorbency $Q_{e q}(\mathrm{~g} / \mathrm{g})$ was calculated using Eq. 1:

$$
Q_{e q}=\frac{W_{1}-W_{0}}{W_{0}}
$$

where $W_{1}(\mathrm{~g})$ and $W_{0}(\mathrm{~g})$ represented the weight of swollen and dried samples, respectively. $Q_{e q}(\mathrm{~g} / \mathrm{g})$ indicated the weight of absorbed solution.
In order to study the effect of $\mathrm{pH}$ on the water absorbency of samples, the different $\mathrm{pH}$ solutions were used as soaking media. Various $\mathrm{pH}$ solutions were prepared by $\mathrm{NaOH}(0.1 \mathrm{~mol} / \mathrm{L})$ and $\mathrm{HCl}(0.1 \mathrm{~mol} / \mathrm{L})$ aqueous solutions. The $Q_{e q}$ of samples in different $\mathrm{pH}$ solutions was determined by the same method.

To study the swelling kinetics of samples in various saline solutions, $10 \mathrm{mmol} / \mathrm{L} \mathrm{NaCl}, \mathrm{CaCl}_{2}, \mathrm{KCl}, \mathrm{MgCl}_{2}$, and $\mathrm{NH}_{4} \mathrm{Cl}$ solutions were prepared, respectively. Specific steps were as follows: $0.5 \mathrm{~g}$ of dried samples were put in nylon bags, which were immersed in the different salt solutions. 


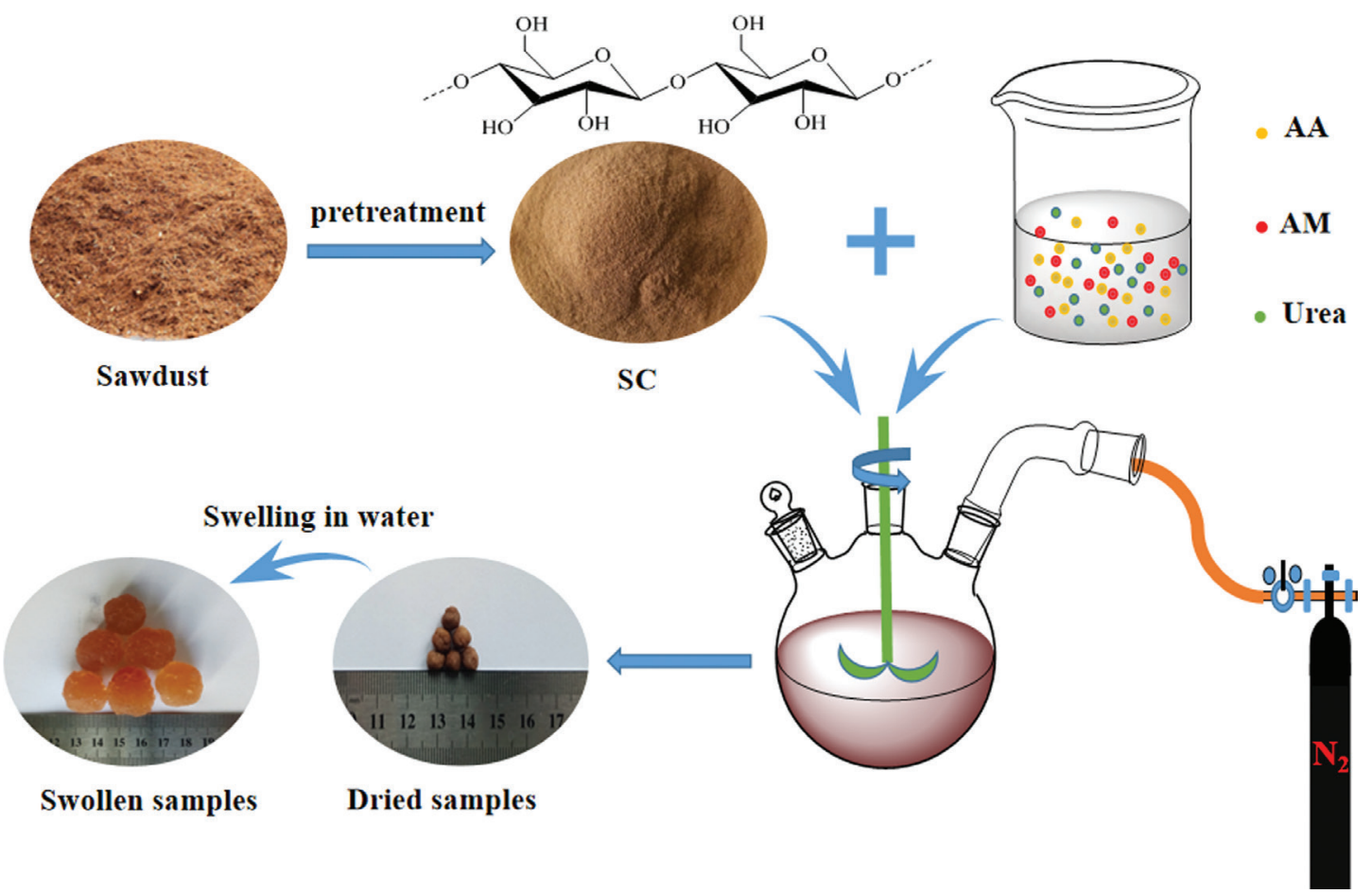

Scheme 2: Diagram of the fabrication process of SC-g-PAA/PAM/Urea.

The bags were separated from the solutions and weighed at prescribed time intervals $(1,3,5,10,15,20,30,45,60$, $90,120,180,240,360,480$, and $600 \mathrm{~min}$ ). The $Q_{t}$ (the water absorbency of samples at time $t$ ) can be calculated by the mentioned equation.

In order to further study the swelling kinetics of samples in water, schott's second-order swelling kinetics model can be indicated by the following equation:

$$
\frac{t}{Q_{t}}=\frac{l}{K_{i s}}=\frac{l}{Q_{m}} t
$$

where $Q_{t}(\mathrm{~g} / \mathrm{g})$ was the water absorbency at any time $t$; $Q_{m}(\mathrm{~g} / \mathrm{g})$ was the theoretical equilibrium water absorbency; $K_{i s}$ was the swelling rate constant.

\subsection{Measurement of the water-holding of SC-g-PAA/PAM/Urea in soil}

1, 2, 3, $4 \mathrm{~g}$ SC-g-PAA/PAM/Urea were mixed with $200 \mathrm{~g}$ of dry soil (20 mesh, the ratio of soil to sand was approximately 9:1) and placed in a $4.5 \mathrm{~cm}$ diameter polyvinyl chloride (PVC) tube, respectively. The bottom of the tube was sealed with nylon mesh and weighed $\left(\mathrm{W}_{0}\right)$. The tap water was poured slowly into the soil from the top of the tube until the water seeped out from the bottom and weighed $\left(\mathrm{W}_{1}\right)$. A control experiment without the SC-g-PAA/PAM/Urea was prepared by the same method. The water holding (WH\%) in the soil was calculated using the following equation:

$$
W H \%=\frac{W_{1}-W_{0}}{W_{0}} \times 100
$$

\subsection{Release behavior and kinetics of SC-g-PAA/PAM/Urea in water}

$1 \mathrm{~g}$ of SC-g-PAA/PAM/Urea was placed in a nylon mesh bag and immersed in $200 \mathrm{~mL}$ of deionized water. The bags were removed from the glass bottle within the specified time intervals $(1,3,5,10,15,20,30,45,60,90,120,180$, 240, 360, and $480 \mathrm{~min}$ ). Approximately $2 \mathrm{~mL}$ of solution was drawn out from the glass bottle to detect the nutrient release of nitrogen using a UV-2450 spectrophotometer. All samples were measured in triplicate, and the results were the average value for each sample.

The $\mathrm{N}$ contentration was calculated using the paradimethyl-amino-benzaldehyde colorimetry. First, $2 \mathrm{~mL}$ fertilizer extract solution was placed in a $25 \mathrm{~mL}$ colorimetric tube. Then, $20 \mathrm{~mL}$ of the $20 \mathrm{~g} / \mathrm{L}$ para-dimethyl-aminobenzaldehyde solution and $2 \mathrm{~mL}$ of the $2 \mathrm{~mol} / \mathrm{L} \mathrm{H}_{2} \mathrm{SO}_{4}$ solution were added. At last, $1 \mathrm{~mL}$ deionized water was added into the solution. The colorimetric tube was shaken to make solution even, and allow to stand for 15 min using 
the spectrophotometer at $422 \mathrm{~nm}$ wavelength to determine $\mathrm{N}$ contentration [18].

In order to further study the release kinetics and mechanism of HSRF in water, Ritger-Peppas release model was employed.

$$
\frac{M_{t}}{M_{\infty}}=K t^{n}
$$

where $M_{t}$ and $M_{\infty}$ were the cumulative fertilizer nutrients release at time $t$ and infinite time, $K$ was the release rate constant, $t$ was the release time and $n$ was the diffusional exponent. When $n \leq 0.45$, the release mechanism belongs to Fick diffusion; when $0.45<n<0.89$, for anomalous transport; when $n \geq 0.89$, it indicates the Case II release mechanism, erosion mechanism for the skeleton $[8,30,31]$. The equation is suitable for the first $60 \%$ of the fractional nutrient release curves [32].

\section{Results and discussion}

\subsection{FTIR spectra}

The FTIR spectra of the SC, AA, AM, urea and SC-g-PAA/ $\mathrm{PAM} /$ Urea were displayed in Figure 1. For the spectrum of
SC, the characteristic peaks at 3426 and $2923 \mathrm{~cm}^{-1}$ were contributed to the $-\mathrm{OH}$ and $\mathrm{C}-\mathrm{H}$ stretching vibration, respectively. And the peaks at 1386 and $1039 \mathrm{~cm}^{-1}$ were attributed to $\mathrm{C}-\mathrm{C}$ and $\mathrm{C}-\mathrm{O}-\mathrm{C}$ stretching vibration. For the spectrum of AA, the intense bands were observed at 1715 and $1627 \mathrm{~cm}^{-1}$, corresponding to the $\mathrm{C}=0$ stretching of $-\mathrm{COOH}$ and $\mathrm{C}=\mathrm{C}$ groups, respectively. And there was a peak at $1434 \mathrm{~cm}^{-1}$, which was assigned to symmetric stretching vibration of $-\mathrm{COO}^{-}$groups [33,34]. The peaks at 1289 and $1238 \mathrm{~cm}^{-1}$ were due to in plane bending vibration of $\mathrm{C}-\mathrm{H}$, while 982 and $812 \mathrm{~cm}^{-1}$ were due to out of plane bending vibration of $\mathrm{C}-\mathrm{H}$. For urea, the peaks at 3440 and $3342 \mathrm{~cm}^{-1}$ were due to asymmetric and symmetric stretching vibration of $-\mathrm{NH}_{2}$ in $-\mathrm{CONH}_{2}$, respectively. The peaks at 1678 and $562 \mathrm{~cm}^{-1}$ were ascribed to the stretching vibration of $-\mathrm{C}=\mathrm{O}$ in $-\mathrm{CONH}_{2}$ and bending vibration of N-CO-N, respectively. For AM, the peaks at 3360, 3176 and $1678 \mathrm{~cm}^{-1}$, which can assigned to the stretching of the $-\mathrm{NH}_{2}$ and $\mathrm{C}=\mathrm{O}$ groups. The peaks at 1272 and $1135 \mathrm{~cm}^{-1}$ were stretching vibration of $-\mathrm{C}-\mathrm{N}$, and 965 and $812 \mathrm{~cm}^{-1}$ were out of plane bending vibration of $\mathrm{C}-\mathrm{H}$.

In the FTIR spectrum of SC-g-PAA/PAM/Urea, the absorption peaks at $3614 \mathrm{~cm}^{-1}$ (stretching vibration of $-\mathrm{OH}$ ), $1551 \mathrm{~cm}^{-1}$ (asymmetric stretching vibration of $-\mathrm{COO}^{-}$ groups), $1410 \mathrm{~cm}^{-1}$ (symmetric stretching vibration of $-\mathrm{COO}^{-}$ groups), $1067 \mathrm{~cm}^{-1}$ (stretching vibration of $\mathrm{C}-\mathrm{O}-\mathrm{C}$ groups) were observed. Moreover, the $\mathrm{C}=\mathrm{O}$ stretching of urea and

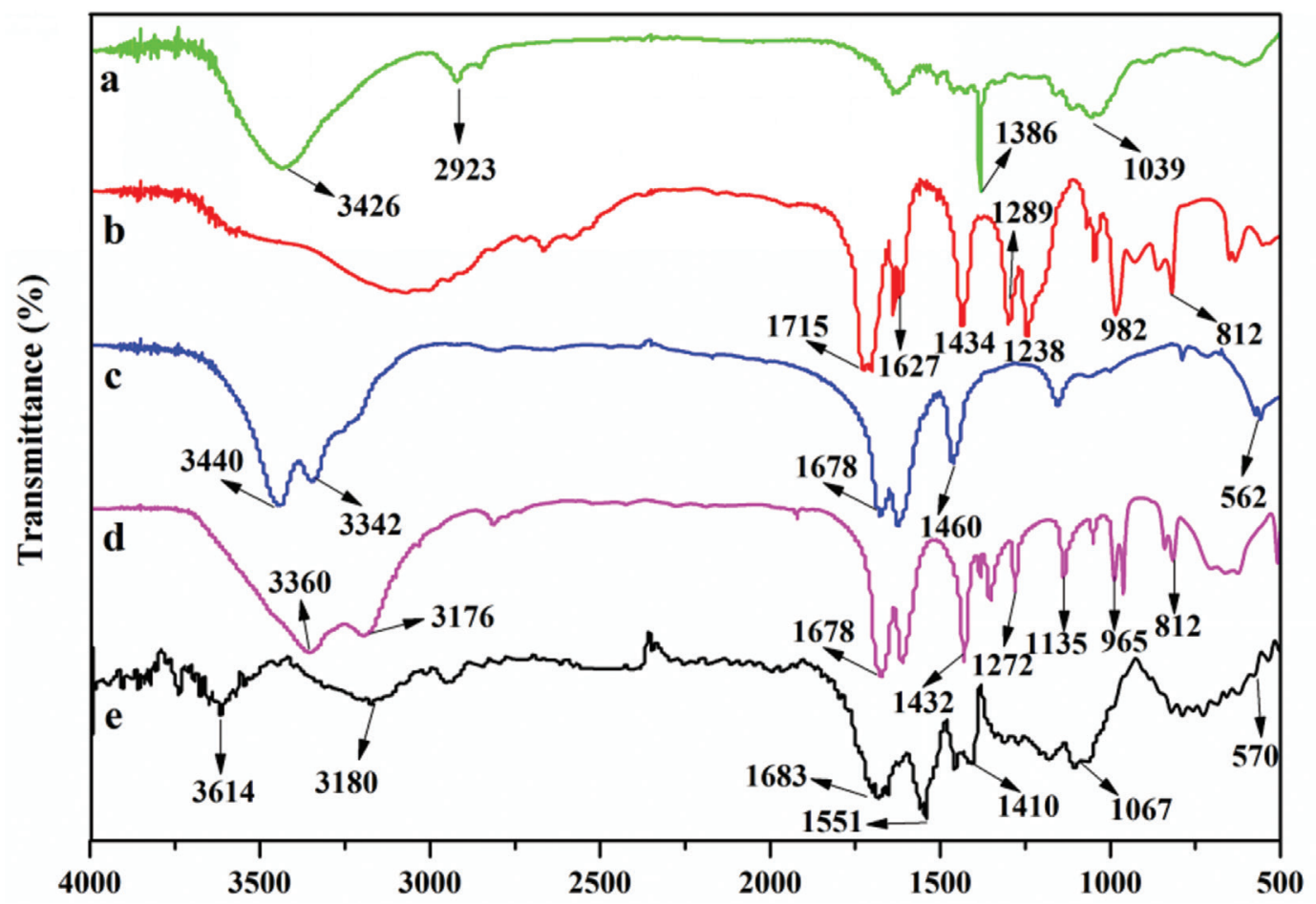

Figure 1: FTIR spectra of SC (a), AA (b), urea (c), AM (d) and SC-g-PAA/PAM/Urea (e). 
$\mathrm{AM}$ at $1678 \mathrm{~cm}^{-1}$ and the $\mathrm{C}=\mathrm{O}$ stretching of $-\mathrm{COOH}$ groups of AA at $1715 \mathrm{~cm}^{-1}$ shifted to $1683 \mathrm{~cm}^{-1}$ in the FTIR spectrum of SC-g-PAA/PAM/Urea, signifying that intermolecular hydrogen-bonding interactions were created between $-\mathrm{COOH}$ and $\mathrm{C}=\mathrm{O}$ groups during the polymerization process $[8,23,35]$. In addition, the characteristic urea bands at $3180 \mathrm{~cm}^{-1}$ with slight shifts, which is the stretching vibration of $-\mathrm{NH}_{2}$ in $-\mathrm{CONH}_{2}$, and $570 \mathrm{~cm}^{-1}$ (bending vibration of $\mathrm{N}-\mathrm{CO}-\mathrm{N}$ ) were visible, suggesting the involvement of urea in the SC-g-PAA/PAM/Urea [20,24,36]. The above analysis of FTIR information concluded that SC-g-PAA/PAM/Urea hydrogel fertilizer was successfully synthesized by using simple reactive methods.

\subsection{X-ray diffraction analysis}

X-ray diffraction analysis was intensively applied as a technique for quantifying the crystalline structure of polymer. Figure 2 exhibits the XRD patterns of SC-g-PAA/ $\mathrm{PAM} /$ Urea, SC and urea. For the SC, the peaks at $2 \theta=15.8^{\circ}$, $22.3^{\circ}$ and $2 \theta=34.6^{\circ}$, corresponded to the characteristic crystalline peaks of cellulose I in nature [19]. The urea XRD patterns showed many characteristic reflections at $2 \theta=22.3^{\circ}, 24.6^{\circ}, 29.4^{\circ}, 31.7^{\circ}, 35.5^{\circ}$, and $2 \theta=37.1^{\circ}$, indicating that urea had a crystalline structure [20]. However, unlike urea and SC, SC-g-PAA/PAM/Urea exhibits a broader and larger peak. The absence of characteristic peaks of urea and SC for the SC-g-PAA/PAM/Urea, indicated that urea and SC had a amorphous structure in the hydrogel fertilizer [20,37] Additionally, the hydrogel fertilizer formed a amorphous structure by crosslinking of SC and urea. Therefore, SC and urea were successfully incorporated in the hydrogel fertilizer. Similar phenomena have been observed by wen et al. that reported the corncob-based hydrogel fertilizers [8].

\subsection{XPS analysis}

The chemical environment of carbon, nitrogen and oxygen atoms in urea and SC-g-PAA/PAM/Urea were studied by XPS. The O 1s (532.71 eV), N 1s (397.93 eV) and C 1s $(284.65 \mathrm{eV})$ on the XPS spectrum of SC-g-PAA/PAM/Urea can be observed in subfigures A1-A3 of Figure 3. The 0 1s (529.78 eV), N 1s (399.83 eV) and C 1s (286.89 eV) on the XPS spectrum of urea can be observed in subfigures B1-B3 of Figure 3. The C1s spectra can be fitted with four peaks at banding energies of 284.72, 286.14, 287.91 and $288.83 \mathrm{eV}$. The four peaks originated from $\mathrm{C}-\mathrm{C}, \mathrm{C}-\mathrm{N}, \mathrm{C}=\mathrm{O}$ and $\mathrm{O}-\mathrm{C}=\mathrm{O}$ groups, respectively. Compared with the XPS spectrum of SC-g-PAA/PAM/Urea, the C1s of urea can be fitted to three peaks (subfigure B2 of Figure 3). The peaks at 284.73, 286.66 and $288.78 \mathrm{eV}$ were attributed to $\mathrm{C}-\mathrm{C}, \mathrm{C}-\mathrm{N}$ and $\mathrm{C}=\mathrm{O}$, respectively. The binding energy and atomic percentage were depicted in Table 1 . Notably, the carbon

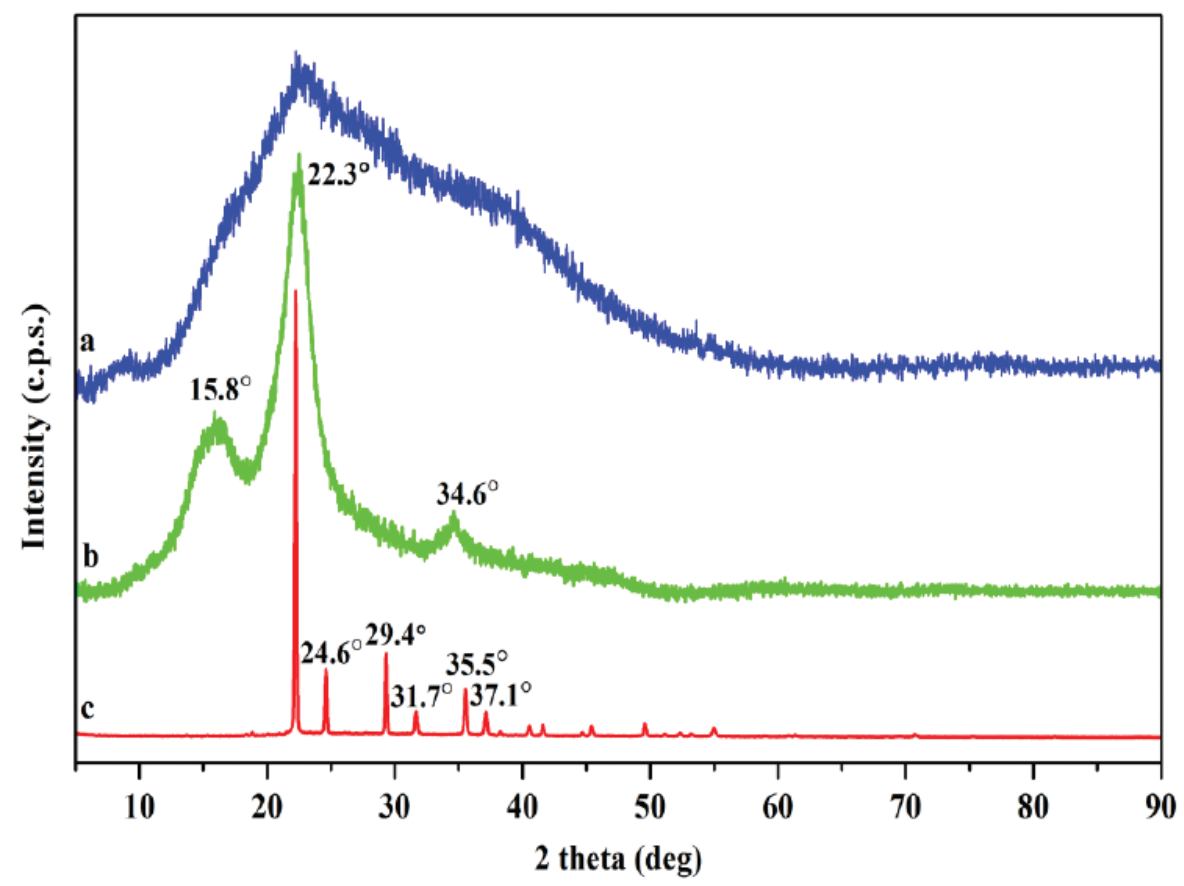

Figure 2: X-ray diffraction patterns of SC-g-PAA/PAM/Urea (a), SC (b) and urea (c). 

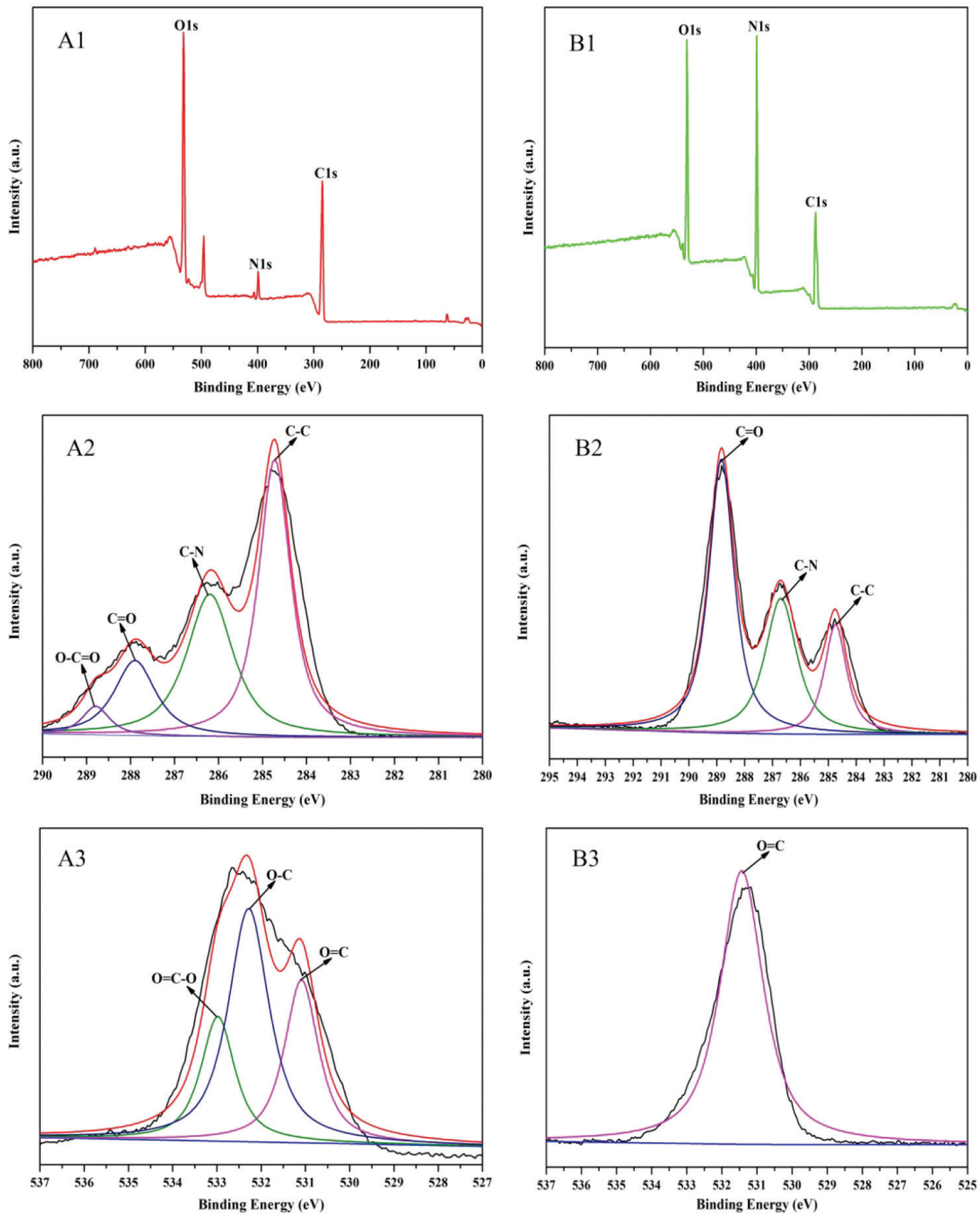

Figure 3: XPS survey spectra of SC-g-PAA/PAM/Urea (A1) and urea (B1), C1s of SC-g-PAA/PAM/Urea (A2), C1s of urea (B2), 01s of SC-g-PAA/ PAM/Urea (A3), 01s of urea (B3).

atomic concentration and oxygen atomic concentration was increased significantly in the SC-g-PAA/PAM/Urea compared with urea. This change was attributed to the large amount of oxygen atoms and carbon atoms in the sawdust cellulose, and sawdust cellulose was successfully incorporated into the SC-g-PAA/PAM/Urea system.

\subsection{Micro-morphology}

The surface elemental composition and distribution of SC-g-PAA/PAM/Urea were displayed in the EDS spectra (Figure 4a) and map (Figure 4c). Table 2 displays the quantitative analysis of the elements present in the EDS 
map. For the surfaces of SC-g-PAA/PAM/Urea, the uniform distributions of $\mathrm{C}, \mathrm{N}, \mathrm{O}, \mathrm{Na}, \mathrm{K}$ and $\mathrm{P}$ elements can be clearly observed. Furthermore, the abundant elements included by the SC-g-PAA/PAM/Urea could be applied to soil and then promoted to improve soil fertility and plant growth [8].

The surface microscopic morphology of SC and SC-g-PAA/PAM/Urea were observed in Figure 5. The SC exhibits block or rod-like morphology. Compared with that of SC, the coarse and fluffy surface was observed

Table 1: XPS analysis of urea and SC-g-PAA/PAM/Urea.

\begin{tabular}{|c|c|c|c|c|c|}
\hline \multicolumn{3}{|c|}{ SC-g-PAA/PAM/Urea } & \multicolumn{3}{|c|}{ Urea } \\
\hline Linkage & $\begin{array}{c}\text { Binding } \\
\text { energy } \\
\text { (eV) }\end{array}$ & $\begin{array}{c}\text { Atomic } \\
\%\end{array}$ & Linkage & $\begin{array}{c}\text { Binding } \\
\text { energy } \\
\text { (eV) }\end{array}$ & $\begin{array}{c}\text { Atomic } \\
\%\end{array}$ \\
\hline $\mathrm{C}-\mathrm{C}$ & 284.72 & 26.23 & $\mathrm{C}-\mathrm{C}$ & 284.73 & 7.78 \\
\hline $\mathrm{C}-\mathrm{N}$ & 286.14 & 18.37 & $\mathrm{C}-\mathrm{N}$ & 286.66 & 8.96 \\
\hline $\mathrm{C}-\mathrm{O}$ & 287.91 & 11.22 & $C=0$ & 288.78 & 12.25 \\
\hline $0-C=0$ & 288.83 & 3.23 & & & \\
\hline $\mathrm{N}-\mathrm{C}$ & 399.47 & 8.26 & $\mathrm{~N}-\mathrm{C}$ & 399.42 & 47.16 \\
\hline $\mathrm{O}=\mathrm{C}$ & 531.05 & 10.73 & $\mathrm{O}=\mathrm{C}$ & 531.42 & 23.85 \\
\hline $0-\mathrm{C}$ & 532.31 & 14.31 & & & \\
\hline $\mathrm{O}=\mathrm{C}-\mathrm{O}$ & 532.96 & 7.65 & & & \\
\hline
\end{tabular}
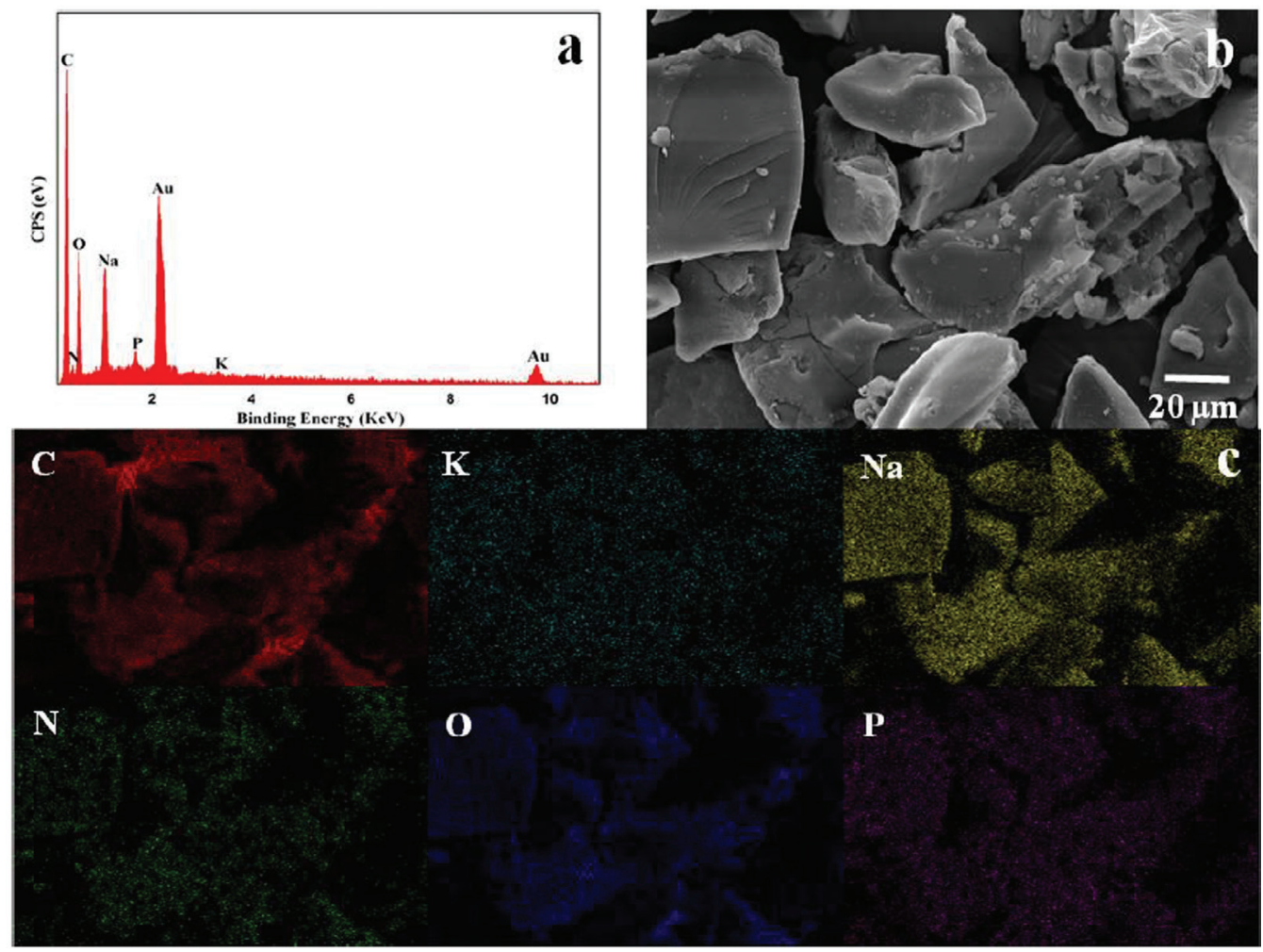

Figure 4: EDS spectra (a) and EDS maps (c) corresponding to the SEM image (b) for surface elemental composition and distribution of SC-g-PAA/PAM/Urea. in the micrograph of SC-g-PAA/PAM/Urea. Additionally, few urea crystals (white spot) were dispersed on the surface of SC-g-PAA/PAM/Urea in Figure 5b. Similar phenomena have been reported by other researchers [20,34].

\subsection{TGA analysis}

TGA was conducted in order to evaluate the thermal stability property of different hydrogel fertilizers in Figure 6. TGA curves of different hydrogel fertilizers exhibit the obvious differences at elevated temperature. The TGA curves of SC-g-PAA/PAM/Urea shows three main thermal degradation stages. The first stage from $200^{\circ} \mathrm{C}$ to $320^{\circ} \mathrm{C}$, it corresponded to the disintegration of branches or side chain groups of the polymer $[36,38,39]$. The second weight loss occurred within $320-520^{\circ} \mathrm{C}$, it could be attributed to the decomposition of main chains of graft copolymer. Finally, the weight loss took place in the range of $520-800^{\circ} \mathrm{C}$, it may be the further decomposition of residual organic matter [38,39]. SC-g-PAA/PAM, SC-g-PAA/Urea and PAA/PAM/Urea shows four main thermal degradation stages, respectively. Notably, the SC-g-PAA/PAM/Urea

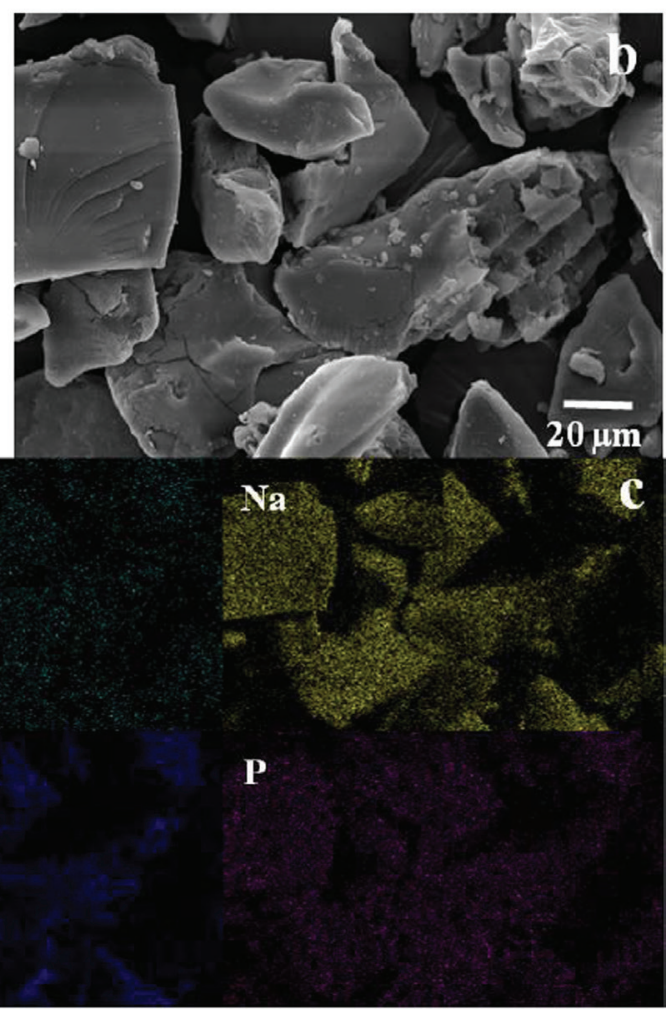


had higher thermal stability than SC-g-PAA/PAM, SC-g$\mathrm{PAA} /$ Urea and PAA/PAM/Urea. When the temperature was $550^{\circ} \mathrm{C}$, the mass ratios of PAA/PAM/Urea, SC-gPAA/Urea, SC-g-PAA/PAM and SC-g-PAA/PAM/Urea were $10.3 \%, 26.7 \%, 37.7 \%, 41.2 \%$, respectively. Similar phenomena have been reported by Wen et al. [8]. Table 3 exhibits the main thermal properties for SC-g-PAA/PAM/ Urea and SC-g-PAA/PAM, SC-g-PAA/Urea and PAA/PAM/ Urea. It was noted that the $5 \%$ weight loss temperature $\left(\mathrm{T}_{5 \%}\right), \mathrm{T}_{20 \%}$ and $\mathrm{T}_{50 \%}$ in the SC-g-PAA/PAM/Urea had higher values than those of SC-g-PAA/PAM, SC-g-PAA/ Urea and PAA/PAM/Urea. Due to the introducing of SC, $\mathrm{AA}$, urea, and AM in hydrogel system, chemical reactions among the molecular chains leaded to the formation of the highly dense network structure in hydrogel, then improving the thermal property of SC-g-PAA/PAM/Urea.

\subsection{Water-holding capacity of soil with SC-g-PAA/PAM/Urea}

The water-holding capacity of soil with SC-g-PAA/PAM/ Urea was displayed in Figure 7. For the soils mixed with

Table 2: Weight percent and atomic percentage of each element in SC-g-PAA/PAM/Urea.

\begin{tabular}{lcc}
\hline Element & wt\% & at\% \\
\hline CK & 40.92 & 50.31 \\
KK & 01.24 & 00.47 \\
NaK & 18.41 & 11.82 \\
NK & 08.36 & 08.82 \\
OK & 30.84 & 28.47 \\
PK & 00.23 & 00.11 \\
\hline
\end{tabular}
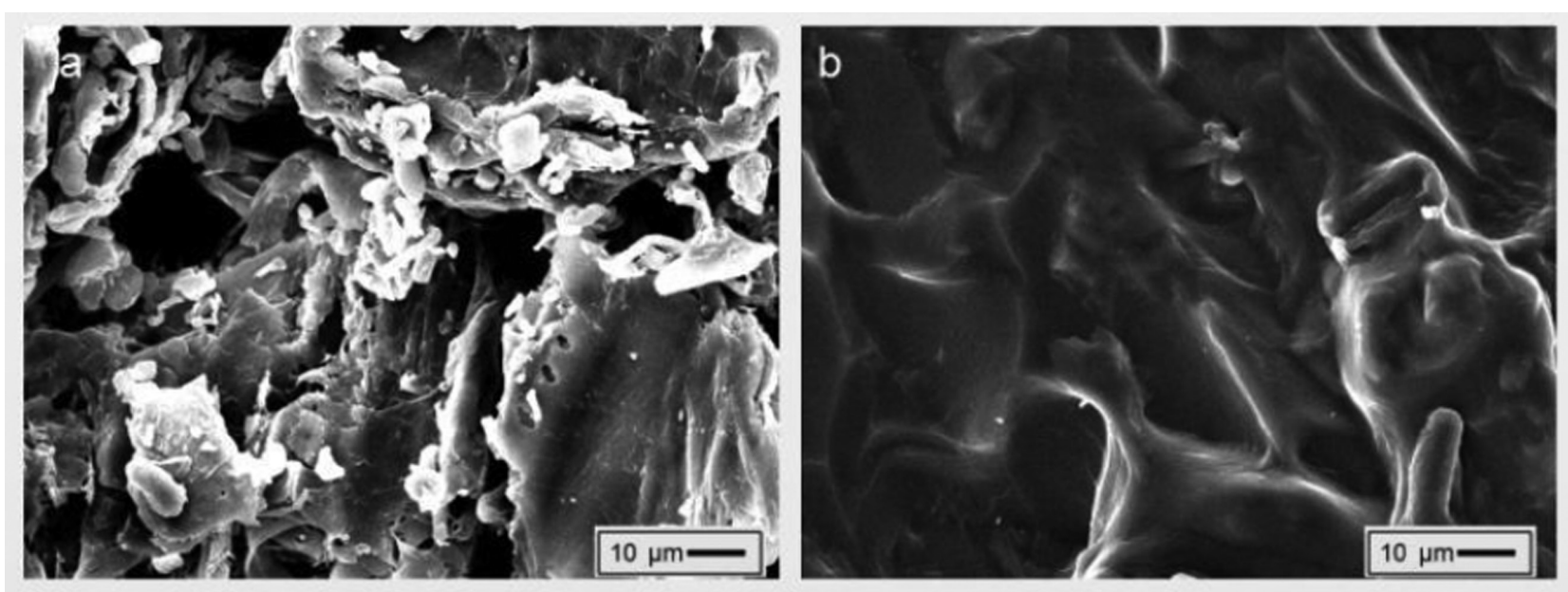

different contents SC-g-PAA/PAM/Urea, soil mixed with $4 \mathrm{~g}$ samples had the largest water-holding ratios (161.2\%), which was higher $122.5 \%$ than that of the soil without hydrogel sample (38.7\%). It indicated that SC-gPAA/PAM/Urea had excellent water-holding capacity. The reason can be that the SC, AA and AM formed skeleton structure by polymerization [40,41]. And the introduction of the SC added the reactive molecular chains length, then increasing water absorption. Simultaneously, the incorporation of SC increased reactive nodes, easily forming skeleton structure. Therefore, the addition of SC can improve water-holding capacity of sample. Furthermore, It was observed that the maximum water-holding capacity would increase with the increase of SC-g-PAA/PAM/Urea dosage in the soil. The higher water-holding capacity can be attributed to the presence of a lot of hydrophilic groups in SC-g-PAA/PAM/Urea $[8,41]$. Therefore, the SC-g-PAA/ $\mathrm{PAM} /$ Urea was applied to the soil, the soil can hold a lot of water during irrigation or rain periods [40]. As a result, application of SC-g-PAA/PAM/Urea fertilizer can effectively reduce irrigation times and improve soil moisture. Additionally, SC-g-PAA/PAM/Urea also releases nutrients and increases crop yields.

\subsection{Effect of salt solution on water absorbency of SC-g-PAA/PAM/Urea}

The swelling curves of SC-g-PAA/PAM/Urea was displayed Figure 8. As can be seen from the figure, the water absorbency of SC-g-PAA/PAM/Urea in $0.01 \mathrm{~mol} / \mathrm{L} \mathrm{NaCl}$ was $108 \mathrm{~g} / \mathrm{g}$. However, the water absorbency of the SC-gPAA/PAM/Urea in $0.50 \mathrm{~mol} / \mathrm{L} \mathrm{NaCl}$ was only $19.4 \mathrm{~g} / \mathrm{g}$.

Figure 5: SEM images of SC (a) and SC-g-PAA/PAM/Urea (b). 


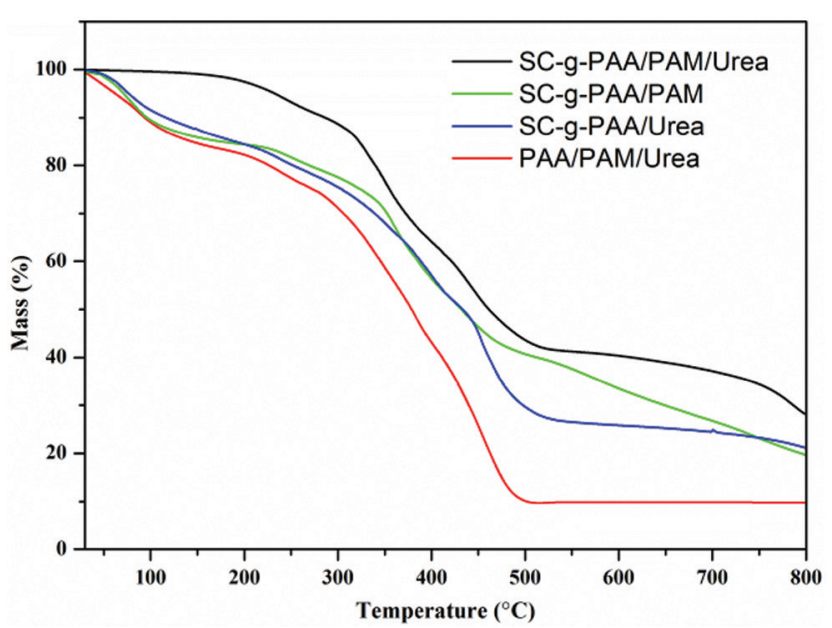

Figure 6: TGA curves of SC-g-PAA/PAM/Urea, SC-g-PAA/PAM, SC-g-PAA/Urea and PAA/PAM/Urea.

Table 3: Summary of thermal properties of SC-g-PAA/PAM/Urea, SC-g-PAA/PAM, SC-g-PAA /Urea and PAA/PAM/Urea.

\begin{tabular}{lcccc}
\hline Samples & $\begin{array}{c}\mathrm{T}_{5 \%} \\
\left({ }^{\circ} \mathrm{C}\right)\end{array}$ & $\begin{array}{c}\mathrm{T}_{20 \%} \\
\left({ }^{\circ} \mathrm{C}\right)\end{array}$ & $\begin{array}{c}\mathrm{T}_{50 \%} \\
\left({ }^{\circ} \mathrm{C}\right)\end{array}$ & $\begin{array}{c}\text { Residue mass } \\
\text { at 800 }\end{array}$ \\
\hline SC-g-PAA/PAM/ & 222.1 & 337.1 & 457.2 & 28.3 \\
Urea & & & & \\
SC-g-PAA/PAM & 68.7 & 244.1 & 396.5 & 19.7 \\
SC-g-PAA/Urea & 71.2 & 275.4 & 434.2 & 21.5 \\
PAA/PAM/Urea & 82.6 & 301.7 & 464.5 & 9.7 \\
\hline
\end{tabular}

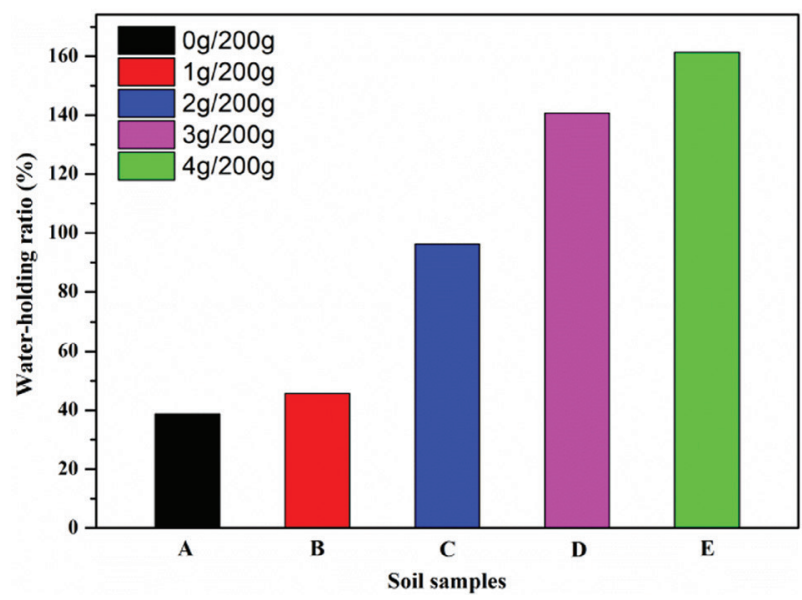

Figure 7: The largest Water-holding ratios of soil samples dosed with different amounts of SC-g-PAA/PAM/Urea.

As evident, the water absorbency of the SC-g-PAA/PAM/ Urea was inversely proportional to the concentration of the sodium chloride solution. This result was attributed to the decreased osmotic pressure difference with increasing saline solution concentration $[8,38,42]$. The osmotic pressure difference acted as a driving force at water absorbency stage of SC-g-PAA/PAM/Urea [42]. The higher concentration of solution would lead to a lower water absorbency driving force [38]. Therefore, SC-g-PAA/PAM/ Urea has strong water absorbency in low concentration $\mathrm{NaCl}$ solution.

\subsection{Swelling capacity and kinetics}

The effects of different saline solutions on the water absorbency of the SC-g-PAA/PAM/Urea were illustrated in Figure 9a. As shown in Figure 9a, the SC-g-PAA/PAM/ Urea had the highest water absorbency in $\mathrm{NaCl}$ solution and the lowest water absorbency in $\mathrm{CaCl}_{2}$ solution. As evident, in the same concentration of salt solution, the water absorbency of the SC-g-PAA/PAM/Urea decreased with the increase of cationic charges. The result was similar to that of LR-PAA/MMT/Urea reported by Zhou et al. [20]. The water absorbency of the SC-g-PAA/PAM/ Urea in $0.01 \mathrm{~mol} / \mathrm{L}$ saline solutions was in the order of $\mathrm{Na}^{+}>\mathrm{K}^{+}>\mathrm{NH}_{4}^{+}>\mathrm{Mg}^{2+}>\mathrm{Ca}^{2+}$. This result was attributed to the formation of complexs by the $-\mathrm{COO}^{-}$groups with multivalent cations, which increased the crosslink density of the hydrogel network [20]. In addition, multivalent cations have higher ionic strength than monovalent cations, so hydrogels had low water absorbency in multivalent cations solutions. Moreover, the smaller radius of the cations were corresponded to the stronger water absorbency capacity of the hydrogel $\left(\mathrm{Na}^{+}>\mathrm{K}^{+}>\mathrm{Mg}^{2+}>\mathrm{Ca}^{2+}\right)$.

Figure $9 \mathrm{~b}$ shows the swelling kinetics of the sample. The $\mathrm{Q}_{m}$ and $\mathrm{K}_{i s}$, and the correlation coefficients $\left(\mathrm{R}^{2}\right)$ were listed in Table 4. The plots revealed lines with good linear correlation coefficient, the $Q_{m}$ of the swelling kinetics model was close to the experimental value, it was indicated that the Schott's second-orderkinetic model can be applied for the swelling process of the SC-g-PAA/PAM/Urea $[17,20,32]$. It can be seen from the figure that the less the water absorbency, the less the $Q_{m}$ value. The swelling rate of the sample was mainly related to the osmotic pressure of the immersed medium and the relaxation rate of the chain segments in the network.

\subsection{Effect of pH on water absorbency of SC-g-PAA/PAM/Urea}

The swelling behavior of the SC-g-PAA/PAM/Urea was investigated in various solutions with different $\mathrm{pH}$ 
values ranging from 2 to 12 . Figure 10 shows the effect of $\mathrm{pH}$ on water absorbency of SC-g-PAA/PAM/Urea. The water absorbency of SC-g-PAA/PAM/Urea increased with the $\mathrm{pH}$ from 2 to 7 . In the case of a solution with a strong acidity most $-\mathrm{COO}^{-}$was protonated and converted to $-\mathrm{COOH}$. In addition, the hydrogen bonds between $-\mathrm{COOH}$ was strengthened by the protonation of $-\mathrm{COO}^{-}$. These results restrained the anion-anion electrostatic repulsion, so decreased the swelling capacity of SC-g-PAA/PAM/Urea at the acidic $\mathrm{pH}[8,17,33]$. With the $\mathrm{pH}$ of the solution increased, some $-\mathrm{COOH}$ groups were ionized and converted to $-\mathrm{COO}^{-}[8]$. At the same time, the hydrogen bonds between $-\mathrm{COOH}$ was weakened, the electrostatic repulsion between $-\mathrm{COO}^{-}$was enhanced, and the water absorbency was increased. At a $\mathrm{pH}$ of 7 , the water absorbency reached a maximum $[8,17,33]$. The water absorbency of the SC-g-PAA/PAM/Urea decreased

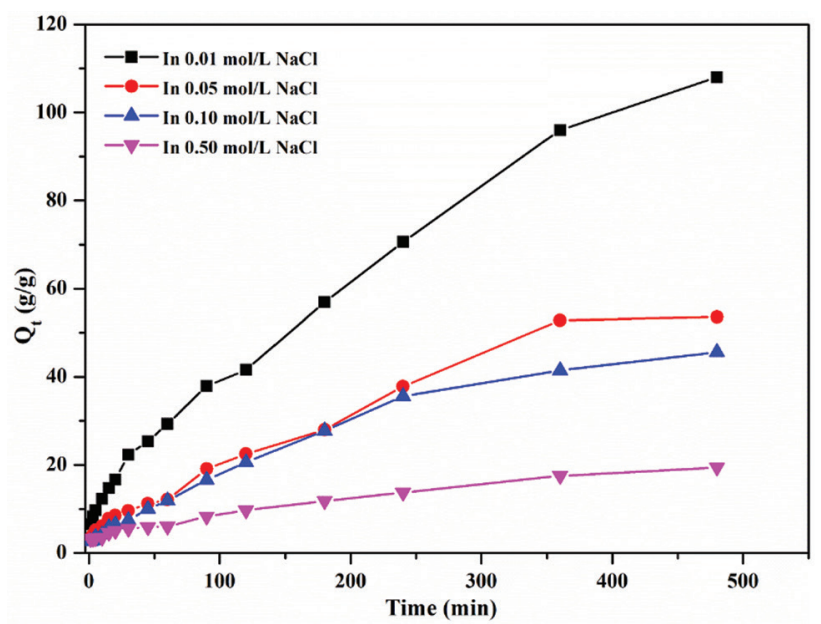

Figure 8: Effect of salt solution on water absorbency of SC-g-PAA/PAM/Urea.

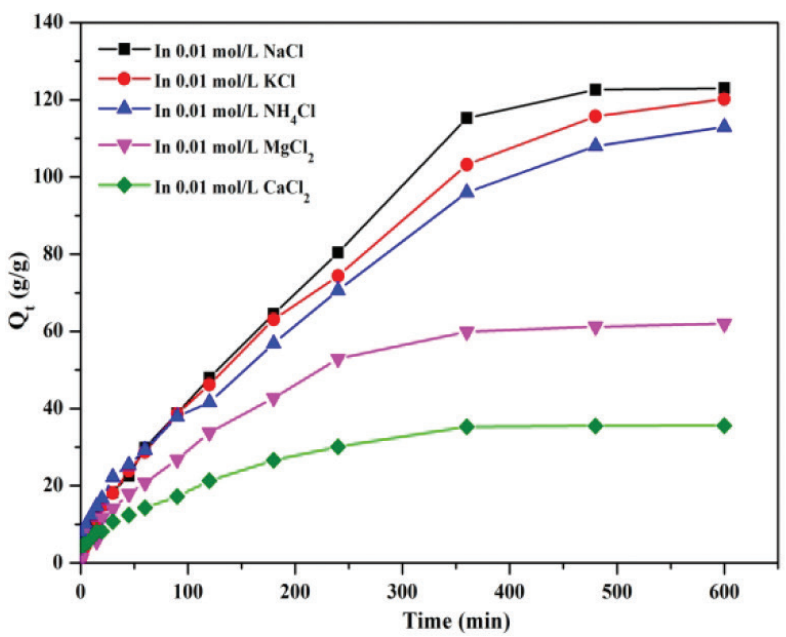

with the $\mathrm{pH}$ from 8 to 12 . This result was attributed to the $\mathrm{Na}^{+}$screening effect. The anion-anion electrostatic repulsion was decreased with the alkalinity of the solution increase and the concentration increase of $\mathrm{Na}^{+}[43,44]$. Therefore, the water absorbency of the SC-g-PAA/PAM/ Urea was reduced.

Table 4: Swelling kinetic parameters of SC-g-PAA/PAM/Urea in different salt solutions.

\begin{tabular}{lccc}
\hline Salt solutions & $\mathbf{K}_{\text {is }}$ & $\mathbf{Q}_{m}$ & $\mathbf{R}^{\mathbf{2}}$ \\
\hline $0.01 \mathrm{~mol} / \mathrm{L} \mathrm{NaCl}$ & 0.0125 & 131.2505 & 0.9200 \\
$0.01 \mathrm{~mol} / \mathrm{L} \mathrm{KCl}$ & 0.0147 & 125.8703 & 0.9710 \\
$0.01 \mathrm{~mol} / \mathrm{L} \mathrm{NH}_{4} \mathrm{Cl}$ & 0.0168 & 114.9425 & 0.9248 \\
$0.01 \mathrm{~mol} / \mathrm{L} \mathrm{MgCl}_{2}$ & 0.0106 & 71.7943 & 0.9598 \\
$0.01 \mathrm{~mol} / \mathrm{L} \mathrm{CaCl}_{2}$ & 0.0098 & 39.4015 & 0.9108 \\
\hline
\end{tabular}

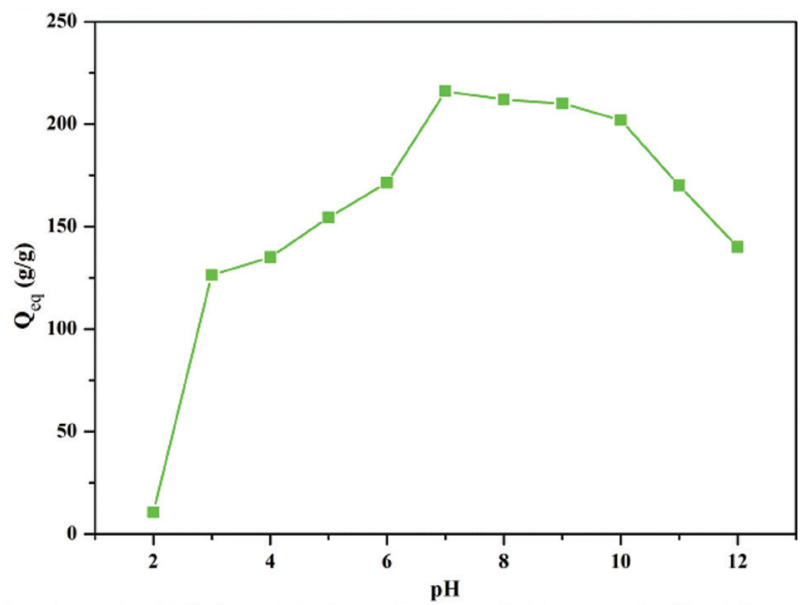

Figure 10: Effect of $\mathrm{pH}$ on water absorbency of SC-g-PAA/PAM/Urea.

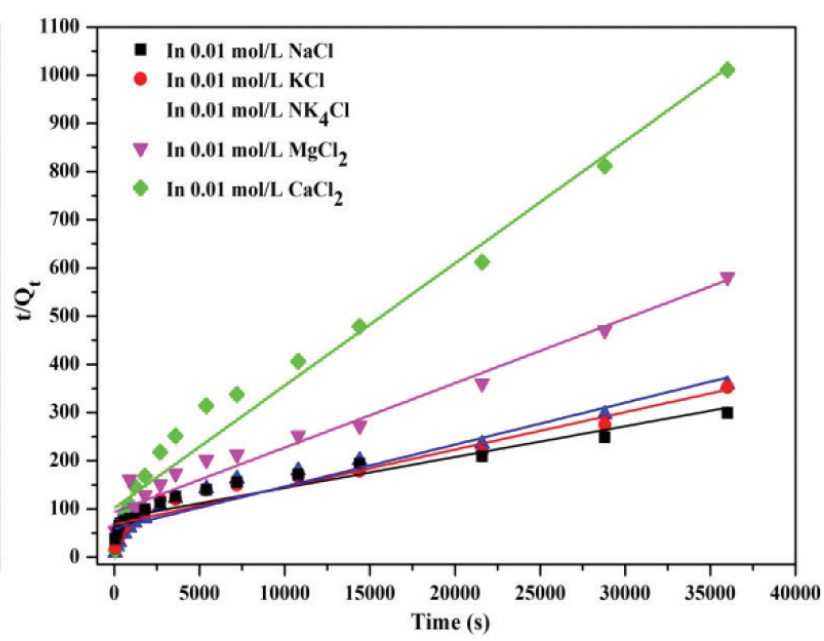

Figure 9: The swelling capacity (a) and kinetics (b) of SC-g-PAA/PAM/Urea in different saline solution. 

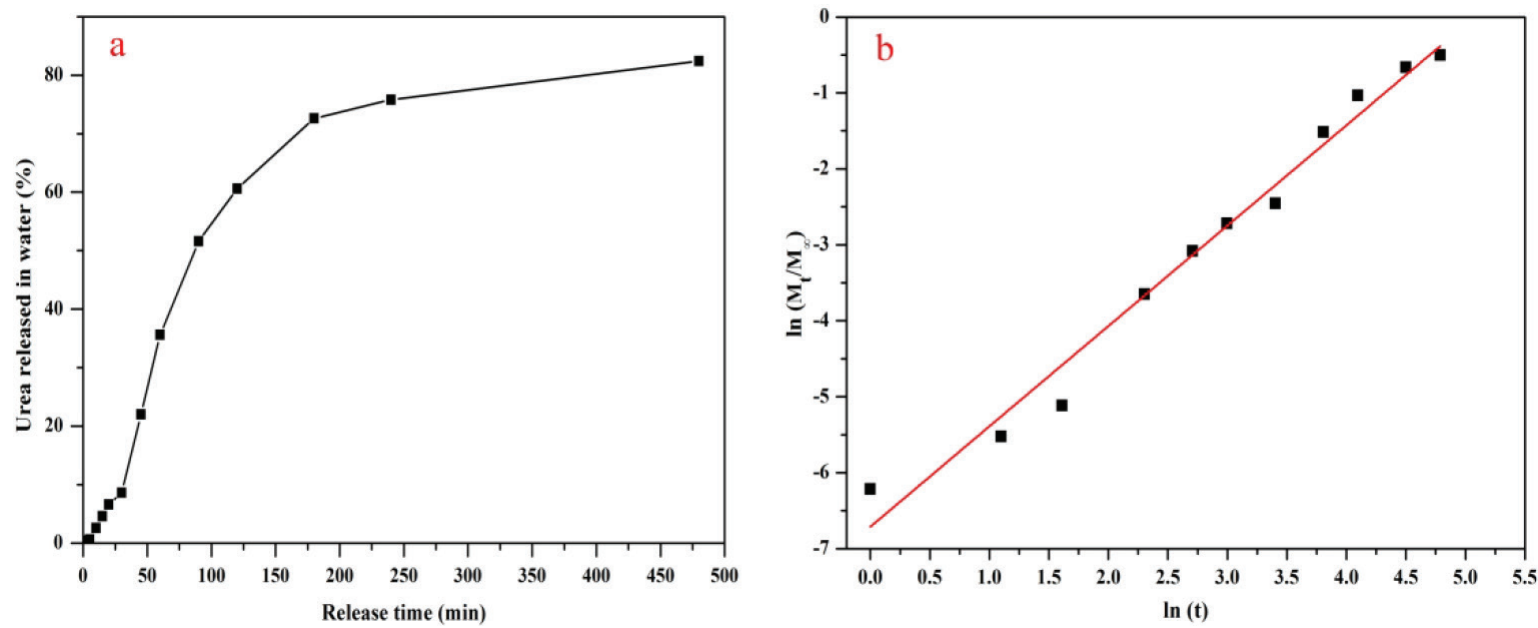

Figure 11: The release behavior (a) and release kinetic (b) of SC-g-PAA/PAM/Urea in water.

Table 5: Release kinetic parameters of SC-g-PAA/PAM/Urea in water.

\begin{tabular}{llccc}
\hline Stages & Equations & $\mathbf{n}$ & $\mathbf{K}$ & $\mathbf{R}^{\mathbf{2}}$ \\
\hline SC-g-PAA/ & $\mathrm{Y}=1.3214 \mathrm{X}-$ & 1.3214 & 0.0012 & 0.9781 \\
PAM/Urea & 6.7114 & & & \\
\hline
\end{tabular}

\subsection{Release behavior and kinetics of SC-g-PAA/PAM/Urea in water}

Figure 11a indicates nutrient release ability of SC-g-PAA/ $\mathrm{PAM} /$ Urea at $25^{\circ} \mathrm{C}$ in water. The cumulative nutrient release of SC-g-PAA/PAM/Urea was 2.6\%, 8.6\%, and $82.4 \%$ within 10, 30, and $480 \mathrm{~min}$, respectively. It could be found that the initial release rate of nitrogen was slow, and then gradually increased. Finally, the nutrient release rate tends to be gentle. This phenomenon could be related with the gel strength of hydrogel network. With the decrease of gel strength, the nutrient release rate gradually increased. Figure 11b shows the release kinetics of SC-g-PAA/PAM/ Urea in water. As can be seen from Table 5 , the $\mathrm{n}$ value in the release model was 1.3214, indicating the Case II release, erosion mechanism for the skeleton. In addition, the curve has a high goodness of fit, indicating that the Ritger-Peppas release model was applicable to explain the diffusional process $[8,30,31]$. The release mechanism was characterized by slow release in the early stage, fast release in the middle stage and slow release in the late stag, which was in line with the growth requirements of the crop.

\section{Conclusions}

Through the method of grafting copolymerization, a novel slow-release hydrogel fertilizer based on sawdust cellulose was successfully prepared. The AA, AM and sawdust cellulose participated in the formation of hydrogel networks in this process, significantly improved the mechanical strength of the hydrogel networks. The effect of reaction variables on the swelling behavior was analyzed. The reaction conditions were optimized to obtain the maximum water absorbency of $210 \mathrm{~g} / \mathrm{g}$ in deionized water. In addition, the influences of salt solutions, ionic strength and solution $\mathrm{pH}$ on the swelling of the SC-g-PAA/PAM/Urea were investigated. SC-g-PAA/ $\mathrm{PAM} /$ Urea can absorb more water in the $\mathrm{pH}$ range $7-10$. Compared to other salt solutions, SC-g-PAA/PAM/Urea was able to absorb more water in $\mathrm{NaCl}$ solution. The release behavior of fertilizer was analyzed using the Ritger-Peppas release model. The addition of SC-g-PAA/ $\mathrm{PAM} /$ Urea into soil could improve the water-retaining ability and reduce nutrient release ability. Therefore, the fertilizer has broad application prospects in agricultural production.

Acknowledgements: This research was funded by the National Key R\&D Program of China (grant no. 2017YFD0200706).

\section{References}

[1] Madusanka N., Sandaruwan C., Kottegoda N., Sirisena D., Munaweera I., De Alwis A., Urea-hydroxyapatite-montmorillonite nanohybrid composites as slow release nitrogen compositions. Appl. Clay Sci., 2017, 150, 303-308.

[2] Chen J., Lu S., Zhang Z., Zhao X., Li X., Ning P., Environmentally friendly fertilizers: $A$ review of materials used and their effects on the environment. Sci. Total Environ., 2018, 613-614, 829-839. 
[3] Liang R., Liu M., Wu L., Controlled release NPK compound fertilizer with the function of water retention. React. Funct. Polym., 2007, 67, 769-779.

[4] Wen P., Wu Z., Han Y., Cravotto G., Wang J., Ye B.C., MicrowaveAssisted Synthesis of a Novel Biochar-Based Slow-Release Nitrogen Fertilizer with Enhanced Water-Retention Capacity. ACS Sustain. Chem. Eng., 2017, 5, 7374-7382.

[5] Zhang, S., Yang, Y., Gao B., Wan Y., Li Y.C., Zhao C., Bio-based Interpenetrating Network Polymer Composites from Locust Sawdust as Coating Material for Environmentally Friendly Controlled-Release Urea Fertilizers. J. Agric. Food Chem., 2016, 64, 5692-5700.

[6] Xie J., Yang, Y., Gao B., Wan Y., Li Y.C., Cheng D., et al., MagneticSensitive Nanoparticle Self-Assembled Superhydrophobic Biopolymer-Coated Slow-Release Fertilizer: Fabrication, Enhanced Performance, and Mechanism. ACS Nano, 2019, 13, 3320-3333.

[7] Chen J., Lü S., Zhang Z., Zhao X., Li X., Ning P., et al., Environmentally friendly fertilizers: A review of materials used and their effects on the environment. Sci. Total Environ., 2018, 613-614, 829-839.

[8] Wen P., Han Y., Wu Z., He Y., Ye B.C., Wang J., Rapid synthesis of a corncob-based semi-interpenetrating polymer network slow-release nitrogen fertilizer by microwave irradiation to control water and nutrient losses. Arabian J. Chem., 2017, 10, 922-934.

[9] Wu F., Zhang Y., Liu L., Yao J., Synthesis and characterization of a novel cellulose-g-poly(acrylic acid-co-acrylamide) superabsorbent composite based on flax yarn waste. Carbohydr. Polym., 2012, 87, 2519-2525.

[10] Zonatto F., Muniz E. C., Tambourgi E.B., Paulino A.T., Adsorption and controlled release of potassium, phosphate and ammonia from modified Arabic gum-based hydrogel. Int. J. Biol. Macromol., 2017, 105, 363-369.

[11] Hüttermann A., Orikiriza L.B., Agaba H., Application of Superabsorbent Polymers for Improving the Ecological Chemistry of Degraded or Polluted Lands. Clean - Soil, Air, Water, 2009, 37, 517-526.

[12] Liu X., Yang Y., Gao B., Li Y., Wan Y., Environmentally Friendly Slow-Release Urea Fertilizers Based on Waste Frying Oil for Sustained Nutrient Release. ACS Sustain. Chem. Eng., 2017, 5, 6036-6045.

[13] Li X., Li Q., Xu X., Su Y., Yue Q., Gao B., Characterization, swelling and slow-release properties of a new controlled release fertilizer based on wheat straw cellulose hydrogel. J. Taiwan Inst. Chem. Eng., 2016, 60, 564-572.

[14] Kenawy E.R., Azaam M.M., El-nshar E.M., Sodium alginateg-poly(acrylic acid-co-2-hydroxyethyl methacrylate)/ montmorillonite superabsorbent composite: Preparation, swelling investigation and its application as a slow-release fertilizer. Arabian J. Chem., 2019, 12, 847-856.
[15] Wang J., Wang W., Wang A., Synthesis, characterization and swelling behaviors of hydroxyethyl cellulose-g-poly(acrylic acid)/attapulgite superabsorbent composite. Polym. Eng. Sci., 2010, 50, 1019-1027.

[16] Qiao D., Liu H., Yu L., Bao X., Simon G.P. , Petinakis E., et al., Preparation and characterization of slow-release fertilizer encapsulated by starch-based superabsorbent polymer. Carbohydr. Polym., 2016, 147, 146-154.

[17] Li X., Li Q., Su Y., Yue Q., Gao B., A novel wheat straw cellulosebased semi-IPNs superabsorbent with integration of waterretaining and controlled-release fertilizers. J. Taiwan Inst. Chem. Eng., 2015, 55, 170-179.

[18] Xiao X., Yu L., Xie F., Bao X., Liu H., Ji Z., One-step method to prepare starch-based superabsorbent polymer for slow release of fertilizer. Chem. Eng. J., 2017, 309, 607-616.

[19] Dai H., Huang H., Enhanced Swelling and Responsive Properties of Pineapple Peel Carboxymethyl Cellulose-g-poly(acrylic acidco-acrylamide) Superabsorbent Hydrogel by the Introduction of Carclazyte. J. Agric. Food Chem., 2017, 65, 565-574.

[20] Zhou T., Wang Y., Huang S., Zhao Y., Synthesis composite hydrogels from inorganic-organic hybrids based on leftover rice for environment-friendly controlled-release urea fertilizers. Sci. Total Environ., 2018, 615, 422-430.

[21] Feng D., Bai B., Wang H., Suo Y., Novel Fabrication of Biodegradable Superabsorbent Microspheres with Diffusion Barrier through Thermo-Chemical Modification and Their Potential Agriculture Applications for Water Holding and Sustained Release of Fertilizer. J. Agric. Food Chem., 2017, 65, 5896-5907.

[22] Ibrahim S., Nawwar G., Sultan M., Development of bio-based polymeric hydrogel: Green, sustainable and low cost plant fertilizer packaging material. J. Environ. Chem. Eng., 2016, 4, 203-210.

[23] Jin S., Yue G., Feng L., Han Y., Yu X., Zhang Z., Preparation and properties of a coated slow-release and water-retention biuret phosphoramide fertilizer with superabsorbent. J. Agric. Food Chem., 2011, 59, 322-327.

[24] Zhang Y., Liang X., Yang X., Liu H., Yao J. An Eco-Friendly SlowRelease Urea Fertilizer Based on Waste Mulberry Branches for Potential Agriculture and Horticulture Applications. ACS Sustain. Chem. Eng., 2014, 2, 1871-1878.

[25] Senna A.M., Braga do Carmo J., Santana da Silva J.M., Botaro V.R., Synthesis, characterization and application of hydrogel derived from cellulose acetate as a substrate for slowrelease NPK fertilizer and water retention in soil. J. Environ. Chem. Eng., 2015, 3, 996-1002.

[26] Shaheen T.I., Emam H.E., Sono-chemical synthesis of cellulose nanocrystals from wood sawdust using Acid hydrolysis. Int. J. Biol. Macromol., 2018, 107, 1599-1606.

[27] Klemm D., Heublein B., Fink H.P., Bohn A., Cellulose: fascinating biopolymer and sustainable raw material. Angew Chem. Int. Ed. Engl., 2005, 44, 3358-3393. 
[28] Nogi M., Yano H., Transparent Nanocomposites Based on Cellulose Produced by Bacteria Offer Potential Innovation in the Electronics Device Industry. Adv. Mater., 2008, 20, 1849-1852.

[29] Abedi A., Cheng H., Dalai A.K., Effects of Natural Additives on the Properties of Sawdust Fuel Pellets. Ener. Fuel., 2018, 32, 1863-1873.

[30] He Y.H., Wu Z.S., Tu L., Han Y.J., Zhang G.L., Li C., Encapsulation and characterization of slow-release microbial fertilizer from the composites of bentonite and alginate. Appl. Clay Sci., 2015, 109-110, 68-75.

[31] Wu Z.S., He Y.H., Chen L.J., Han Y.J., Li C., Characterization of Raoultella planticola Rs-2 microcapsule prepared with a blend of alginate and starch and its release behavior. Carbohyd. Polym., 2014, 110, 259-267.

[32] Ritger P.L., Peppas N.A., A simple equation for description of solute release 2 . Fickian and anomalous release from swellable devices. J. Control. Release, 1987, 5, 37-42.

[33] Tally M., Atassi Y., Optimized synthesis and swelling properties of a $\mathrm{pH}$-sensitive semi-IPN superabsorbent polymer based on sodium alginate-g-poly(acrylic acid-co-acrylamide) and polyvinylpyrrolidone and obtained via microwave irradiation. J. Polym. Res., 2015, 22, 181-194.

[34] Wen P., Wu Z., He Y., Ye B.C., Han Y., Wang J., Microwave-assisted synthesis of a semi-interpenetrating polymer network slowrelease nitrogen fertilizer with water absorbency from cotton stalks. ACS Sustain. Chem. Eng., 2016, 4, 6572-6579.

[35] Wang W.B., Wang Q., Wang A.Q., PH-responsive carboxy -methylcellulose-g-poly(sodium acrylate)/polyvinylpyrrolidone semi-IPN hydrogels with enhanced responsive and swelling properties. Macromol. Res., 2011, 19, 57-65.

[36] Wen P., Wu Z.S., He Y.H., Han Y.J., Tong Y.B., Characterization of $p(A A-c o-A M) /$ bent/urea and its swelling and slow release behavior in a simulative soil environment. J. Appl. Polym. Sci., 2016, 133, 43082-43093.
[37] Zhou L.L., Cai D.Q., He L.L., Zhong N.Q., Yu M., Zhang X., et al., Fabrication of a high-performance fertilizer to control the loss of water and nutrient using micro/nano networks. ACS Sustain. Chem. Eng., 2015, 3, 645-653.

[38] Zhang M.Y., Cheng Z.Q., Zhao T.Q., Liu M.Z., Hu M.J., Li J.F., Synthesis, characterization, and swelling behaviors of salt sensitive maize bran-poly(acrylic acid) superabsorbent hydrogel. J. Agric. Food Chem., 2014, 62, 8867-8874.

[39] Zhu G.Z., Deng X.L., Hou M., Sun K., Zhang Y.P., Li P., et al., Comparative study on characterization and adsorption properties of activated carbons by phosphoric acid activation from corncob and its acid and alkaline hydrolysis residues. Fuel Process. Technol., 2016, 144, 255-261.

[40] Lü S.Y., Feng C., Gao C.M., Wang X.G., Xu X.B., Bai X., et al., Multifunctional environmental smart fertilizer based on L-aspartic acid for sustained nutrient release. J. Agric. Food Chem., 2016, 64, 4965-4974.

[41] Xie L.H., Liu M.Z., Ni B.L., Wang Y.F., New environment-friendly use of wheat straw in slow-release fertilizer formulations with the function of superabsorbent. Ind. Eng. Chem. Res., 2012, 51, 3855-3862.

[42] Zhou Y.M., Fu S.Y., Zhang L.L., Zhan H.Y., Superabsorbent nanocomposite hydrogels made of carboxylated cellulose nanofibrils and CMC-g-p(AA-co-AM). Carbohyd. Polym., 2013, 97, 429-435.

[43] Rashidzadeh A., Olad A., Slow-released NPK fertilizer encapsulated by NaAlg-g-poly(AA-co-AAm)/MMT superabsorbent nanocomposite. Carbohyd. Polym., 2014, 114, 269-278.

[44] Rashidzadeh A., Olad A., Salari D., Reyhanitabar A., On the preparation and swelling properties of hydrogel nanocomposite based on sodium alginate-g-poly (acrylic acid-co-acrylamide)/ clinoptilolite and its application as slow release fertilizer. J. Polym. Res., 2014, 21, 1-15. 